\title{
Perfil de sensibilización alérgica en inmigrantes residentes en la zona centro de España
}

\section{Allergic sensitization profile in the immigrant population living in the central region of Spain}

\author{
J. Domínguez-Ortega ${ }^{1}$, D. González de Olano $^{2}$, M.J. Trujillo ${ }^{3}$, A. Henríquez ${ }^{4}$, A. Losada ${ }^{5}$, \\ B. Rodríguez-Domínguez ${ }^{6}$, en representación del Grupo ESPLORA*
}

\section{RESUMEN}

Introducción. La prevalencia de las enfermedades alérgicas ha aumentado últimamente, especialmente en países industrializados. Puesto que la alergia está influenciada por factores genéticos y ambientales, la población inmigrante constituye un buen modelo epidemiológico para valorar influencia del ambiente. Presentamos las características clínicas de alergia respiratoria en población inmigrante del centro de España.

Métodos. Se recogieron datos prospectivamente de 62 pacientes inmigrantes y 33 españoles que acudieron en marzo de 2010 a 7 hospitales de Madrid, Cuenca y Ciudad Real, diagnosticados de alergia respiratoria mediante un estudio alergológico estándar.

Resultados. El 63\% eran mujeres, edad media 28,4 años. Sus países de origen se distribuyeron en 3 grupos (Norte de África, Latinoamérica, Europa del Este). El 96\% presentaban rinitis, aunque sólo el $48 \%$ de españoles frente al $76,9 \%$ de hispanos sufrían rinitis persistente. La prevalencia de asma fue similar, aunque entre hispanos el porcentaje de asma controlada fue menor. El tiempo de evolución fue menor en pacientes inmigrantes. El polen de gramíneas resultó el alérgeno más relevante salvo en pacientes árabes. La incidencia de sensibilización a polcalcina fue muy baja. Por el contrario, el $44,1 \%$ de españoles presentaban sensibilización a profilina, frente al 4,88 de hispanos, aunque no implicaba sintomatología al tomar vegetales en la mayoría de casos.

Conclusiones. Este estudio multicéntrico muestra diferencias significativas en tiempo de evolución y perfiles de sensibilización entre pacientes españoles e inmigrantes con alergia respiratoria. Además, diferencias en la expresión clínica de la rinitis y peor control del asma, pueden condicionar peor evolución clínica en el colectivo inmigrante.

Palabras clave. Alergia respiratoria. Alergia. Sensibilización. Inmigrantes. España.

\begin{abstract}
Introduction. The prevalence of allergic diseases has increased worldwide in the last two decades, particularly in developed countries. Respiratory allergy is determined by genetic heredity, influenced by environmental factors. Migration is a good epidemiological model for assessing the influence of the environment. We present the clinical characteristics of respiratory allergy in immigrants in the central region of Spain.
\end{abstract}

Methods. We prospectively collected data on all immigrants referred to the allergy units of 7 different hospitals in Madrid, Cuenca and Ciudad Real in March 2010. Respiratory Allergy was diagnosed using a standard study for allergic diseases. Results in immigrants were compared with data from a similar Spanish population.

Results. Sixty-two immigrants and 32 Spanish patients were evaluated ( $63 \%$ female, mean age 28.4 years). Their countries of origin were uniformly distributed among 3 macroareas (North of Africa, Latin America, and Eastern Europe). More than $96 \%$ presented rhinitis, although persistent rhinitis was more prevalent amongst Latin Americans (76.9\%) than in the Spanish population (48\%). No differences were observed in asthma prevalence, although immigrants had higher rates of non-controlled and partially controlled asthma. The mean time of onset of symptoms after immigration was 43 months. Grass pollen was the most relevant allergen with the exception of Arab patients. Sensitization to polcalcin was rare Otherwise, $44.1 \%$ of the Spanish population was sensitized to profilin (only $4.88 \%$ among immigrants) although this sensitization did not associate allergy to fruits or other vegetables.

Conclusions. In summary, a multicentric study is presented where significant differences have been observed in the sensitization pattern and clinical evolution of respiratory allergy in immigrants and the Spanish population.

Key words. Respiratory allergy. Allergy. Immigrants. Sensitization. Spain.
1. Servicio de Alergología. Hospital Universitario de Getafe. Madrid

2. Servicio de Alergología. Hospital de Fuenlabrada. Madrid

3. Servicio de Alergología. Hospital del Tajo. Aranjuez. Madrid

4. Servicio de Alergología. Hospital Infanta Elena. Valdemoro. Madrid

5. Servicio de Alergología. Hospital Infanta Cristina. Parla. Madrid

6. Servicio de Alergología. Hospital de Tomelloso. Ciudad Real

\section{Correspondencia:}

Javier Domínguez Ortega

Unidad de Alergología

Hospital Universitario de Getafe

Ctra. de Toledo, Km 12,500

28905 Getafe. Madrid. España

E-mail: alergología.hugf@salud.madrid.org

Recepción: 23 de diciembre de 2010

Aceptación provisional: 25 de enero de 2011

Aceptación definitiva: 25 de febrero de 2011 


\section{INTRODUCCIÓN}

Existen evidencias de que la prevalencia de las enfermedades alérgicas ha aumentado en los últimos años en todo el mundo, especialmente en los países industrializados. Es bien conocido que la alergia respiratoria está influenciada por factores genéticos que determinan la susceptibilidad de un individuo, así como por la exposición a factores ambientales. Es particularmente significativo lo ocurrido en Alemania, que con una misma base genética, presentaba grandes diferencias en la prevalencia de enfermedades alérgicas antes de la reunificación, tendiendo a igualarse en estos 20 años en los que el Este del país se ha adaptado al tipo de vida occidental ${ }^{1}$. Que el estilo de vida, la dieta, el padecimiento de infecciones o la contaminación ambiental influyen en el desarrollo de enfermedades alérgicas, está ampliamente documentado ${ }^{2}$. Factores como el aumento de las partículas diesel se han asociado al aumento de sensibilizaciones frente a aero-alérgenos, particularmente pólenes, al facilitar su depósito actuando como portadoras de los mismos en el aire ${ }^{3}$.Así, son notables las diferencias en la prevalencia de enfermedades alérgicas según las distintas regiones del mundo, siendo éstas más altas en los países más desarrollados. Por ejemplo, 150 millones de personas en todo el mundo padecen asma, aunque la prevalencia varía entre el $1 \%$ del área rural de Etiopía y el 25\% de los niños en Australia ${ }^{4}$.

En los últimos 10 años, España ha contemplado un aumento notable del fenómeno de inmigración. La población inmigrante constituye un grupo heterogéneo por su diferente origen, cultura, dieta, hábitos o situación socio-económica. El inmigrante, además, se expone a nuevos alérgenos y factores ambientales diferentes a los de sus países de origen. Por ello, puede ser un buen modelo epidemiológico para valorar la influencia del ambiente en el desarrollo de enfermedades alérgicas. De hecho, se conoce que la tasa de alérgicos en la población inmigrante en países desarrollados es mayor que la observada entre la misma población residente en sus países de origen ${ }^{5}$, aunque genéticamente ambas poblaciones serían similares. La idea del presente estudio es tratar de relacionar el desarrollo de alergia con el tiempo de residencia en un nuevo país, tratando de identificar una posible relación con la exposición a factores ambientales distintos, así como investigar si en los inmigrantes la alergia respiratoria se muestra con una mayor virulencia que en la población autóctona española.

\section{MATERIAL Y MÉTODOS}

Se realizó un estudio descriptivo transversal con todos los pacientes no nacidos en España, incluidos de forma consecutiva, que consultaron durante el mes de marzo de 2010 por posible alergia respiratoria en los servicios y unidades de Alergología de los hospitales de Madrid: Infanta Cristina (Valdemoro), Infanta Elena (Parla), Hospital de Fuenlabrada, Hospital del Tajo (Aranjuez) y Hospital Universitario de Getafe; Hospital de Tomelloso (Ciudad Real) y Hospital Virgen de la Luz (Cuenca). Se consideraron como casos aquellos pacientes nacidos fuera de España, de cualquier edad y etnia, en los que se confirmó el diagnóstico de alergia respiratoria (rinitis, conjuntivitis y/o asma). Se recogió información a través de la historia clínica, considerándose variables demográficas la edad, sexo, país de origen y área de residencia actual (rural, semi urbana o urbana) así como el tiempo, en meses, desde que comenzó a residir en España. Las nacionalidades se agruparon como Europa del Este, África del Norte, África Subsahariana, Asia y Latinoamérica. Como controles, se recogió información, durante el mismo periodo de tiempo en el que se incluyó a la población inmigrante, de pacientes españoles visitados por primera vez e igualmente diagnosticados de alergia respiratoria, hasta alcanzar una proporción 2:1 con respecto al número total de pacientes extranjeros del resto del mundo.

Se consideraba el diagnóstico de rinitis, conjuntivitis y asma por separado y el tiempo en meses desde la aparición de los síntomas, así como la clasificación según 
los criterios de la guía ARIA ${ }^{6}$ para la rinitis, y GEMA $2009^{7}$ (utilizando el Test de Control del Asma $)^{8}$ para el asma, estableciéndose el grado de control del mismo en el momento de la consulta. Se recogían datos de sensibilización alérgica a aeroalérgenos incluidos en una batería estándar común en todos los centros (pólenes: Phleum pratense, Olea europaea, Platanus hispanica, $\mathrm{Cu}$ pressus arizonica, Plantago lanceolata, Artemisia vulgaris, Chenopodium album, Salsola kali; ácaros (Dermatophagoides pteronyssinus y Dermatophagoides farinae; epitelios de perro y gato, Alternaria alternata así como profilina y polcalcina como principales panalérgenos; ALK-Abelló, S.A. Madrid. España.), a través de los resultados de las pruebas cutáneas en Prick. También se consignó la existencia de otras enfermedades alérgicas asociadas (alergia a alimentos, alergia a medicamentos, urticaria, dermatitis alérgica de contacto, dermatitis atópica $o$ alergia a veneno de himenópteros). Finalmente, para valorar influencia genética se consideraba la existencia de antecedentes familiares de alergia entre familiares de primer grado.

Los pacientes eran codificados con un número único, de modo que se garantizara el anonimato fuera del investigador principal de cada centro. El procesamiento de los datos se realizó también de forma anónima.

Para el análisis estadístico se empleó el programa estadístico SAS 9.1. Para las variables cualitativas se presentó la frecuencia absoluta y el porcentaje de individuos en cada categoría. Para las variables cuantitativas se presentaron tablas de es- tadísticos descriptivos como la media, la desviación típica, la mediana, el mínimo y máximo y finalmente el rango intercuartílico de cada variable. La asociación entre las variables cualitativas se realizó mediante el test del chi-cuadrado o mediante la prueba exacta de Fisher cuando las condiciones de aplicación de la prueba chi-cuadrado no se cumplían. En el caso de las variables cuantitativas, se comprobó que no se ajustaban a una distribución normal (prueba Shapiro de normalidad) por lo que las pruebas de igualdad de medias se efectuaron mediante la prueba Wilcoxon (no-paramétrica).

\section{RESULTADOS}

Se recogieron datos de 62 pacientes no nacidos en España y 33 pacientes españoles, diagnosticados de alergia respiratoria en marzo de 2010 en todos los centros implicados en el estudio. Por etnias, 41 pacientes eran hispanos, 10 caucásicos de Europa del Este y 9 árabes (1 paciente subsahariano y 1 asiático se excluyeron del estudio por no poderse incluir en ninguna etnia). El $63 \%$ de toda la muestra eran mujeres, siendo este porcentaje similar en todos los grupos étnicos estudiados (Tabla 1). La edad media en los inmigrantes fue de 29,3 años y en los españoles de 27,8 . El 70,59\% de los españoles y el 80,59 de los hispanos eran no fumadores siendo el porcentaje de fumadores mayor en el de los pacientes del Este (40\%). Mayoritariamente, residían en núcleos urbanos. El porcentaje de pacientes con antecedentes familiares de alergia fue muy semejante en todos los grupos analizados.

Tabla 1. Variables demográficas de los pacientes.

\begin{tabular}{lcclc}
\hline $\begin{array}{c}\text { Nacionalidad } \\
\text { inmigrantes }\end{array}$ & $\begin{array}{c}\text { No.* } \\
\text { (\%) }\end{array}$ & $\begin{array}{c}\text { Hombre/Mujer } \\
\text { \% }\end{array}$ & Área residencia** & Tiempo*** $^{* *}$ \\
\hline Europa Este & $10(17 \%)$ & $50 / 50$ & Urbana $(70 \%)$ & $78(36-128)$ \\
\hline Hispano & $41(68 \%)$ & $32 / 68$ & Urbana $(97,5 \%)$ & $93(10-240)$ \\
\hline Árabe & $9(15 \%)$ & $44 / 56$ & Urbana $(66,6 \%)$ & $115(48-240)$ \\
\hline Españoles & $33(100 \%)$ & $35 / 65$ & Urbana $(100 \%)$ & $159(24-250)$ \\
\hline
\end{tabular}

\footnotetext{
"Número de pacientes expresado en porcentaje respecto del total de inmigrantes.

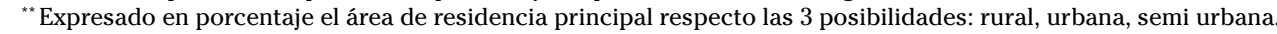

${ }^{* * *}$ Tiempo de residencia en España, expresado en meses (mediana, mínimo-máximo).
} 
El $85,37 \%$ de los hispanos y el $94,12 \%$ de los españoles presentaban conjuntivitis alérgica. Más del 96\% de los pacientes de estos dos grupos presentaban rinitis, si bien sólo el $48 \%$ de los españoles frente al 76,9\% de los hispanos fueron diagnosticados de rinitis persistente según la clasificación ARIA $(\mathrm{p}=0,01)$. El porcentaje de pacientes que además se diagnosticaron de asma en ambos grupos de población fue similar $(69,70$ y 65,85$)$, aunque en el grupo de hispanos el porcentaje de asma parcialmente controlada y no controlada resultó mayor $(52,64 \%)$ frente al $43,58 \%$ en los españoles $(\mathrm{p}<0,05)$. El tiempo medio de residencia en nuestro país se situó alrededor de los 84 meses. A reseñar que el tiempo de evolución para desarrollar conjuntivitis, rinitis y/o asma fue claramente menor en el grupo de pacientes inmigrantes, especialmente entre los hispanos, que el necesario entre los pacientes de origen español (43 meses y 93 respectivamente $(\mathrm{p}<0,001)$ (Tabla 2).

Tabla 2. Clínica referida, intensidad de los síntomas y control de los mismos, según grupos de población por origen.

\begin{tabular}{|c|c|c|c|c|c|}
\hline & $\begin{array}{c}\text { Españoles } \\
(\mathrm{n}=33)\end{array}$ & $\begin{array}{l}\text { Europa Este } \\
\quad(\mathrm{n}=10)\end{array}$ & $\begin{array}{c}\text { Hispanos } \\
(\mathrm{n}=41)\end{array}$ & $\begin{array}{c}\text { Árabes } \\
(\mathrm{n}=9)\end{array}$ & $\mathbf{p}$ \\
\hline \multicolumn{6}{|l|}{ AP alergológicos } \\
\hline RAA & $7(20,5 \%)$ & $0(0 \%)$ & $8(20 \%)$ & $1(11,1 \%)$ & NS \\
\hline SAO & $4(11,7 \%)$ & $0(0 \%)$ & $2(5 \%)$ & $1(11,1 \%)$ & NS \\
\hline $\mathrm{DA}$ & $0(0 \%)$ & $0(0 \%)$ & $1(2.5 \%)$ & $1(11,1 \%)$ & NS \\
\hline RAM & $6(17,6 \%)$ & $3(30 \%)$ & $0(0 \%)$ & $0(0 \%)$ & 0,007 \\
\hline $\mathrm{DC}$ & $1(2,9 \%)$ & $1(10 \%)$ & $0(0 \%)$ & $0(0 \%)$ & NS \\
\hline Rinitis & $32(97 \%)$ & $10(100 \%)$ & $39(95,1 \%)$ & $9(100 \%)$ & NS \\
\hline Intermitente & $17(51,5 \%)$ & $4(40 \%)$ & $9(23 \%)$ & $2(22,2 \%)$ & NS \\
\hline Persistente & $16(48,8 \%)$ & $6(60 \%)$ & $30(76,9 \%)$ & $7(77,7 \%)$ & $0.01^{*}$ \\
\hline Conjuntivitis & $32(94,1 \%)$ & $8(80 \%)$ & $35(85,4 \%)$ & $6(66,6 \%)$ & NS \\
\hline Asma & $23(69,7 \%)$ & $5(50 \%)$ & $27(65,8 \%)$ & $7(77,7 \%)$ & NS \\
\hline Controlada & 13 & 2 & 15 & 2 & NS \\
\hline Parcialmente c. & $10(43,4 \%)^{* *}$ & 2 & $9(33,3 \%)^{* *}$ & 4 & $0,03^{*}$ \\
\hline No controlada & 0 & 1 & $3(11,1 \%)^{* *}$ & 1 & \\
\hline Tiempo síntomas ${ }^{\star \star *}$ & $93(10-240)$ & $57(6-180)$ & $43(12-200)$ & $46(11-264)$ & $0,001^{*}$ \\
\hline
\end{tabular}

AP; antecedentes personales, RAA; reacciones adversas con alimentos, NS; no significación estadística, SAO; síndrome de alergia oral, DA; dermatitis atópica, RAM; reacciones adversas con medicamentos, DC; dermatitis contacto.

"Valor de p comparando pacientes caucásicos frente a hispanos.

${ }^{*}$ Porcentaje sobre el total de asmáticos de cada grupo poblacional.

${ }^{* * *}$ Promedio del tiempo evolución desde inicio de síntomas de rinitis-conjuntivitis-asma, expresado en meses (mediana, mínimo-máximo).

Por sensibilizaciones, tanto en el grupo de pacientes hispanos, en el de pacientes del Este de Europa, y en el de españoles, el polen de gramíneas resultó el alérgeno más relevante $(73,17 \%, 60 \%$ y $82,35 \%$ respecti- vamente). Por el contrario, en el grupo de pacientes árabes, los ácaros del polvo constituyeron los alérgenos más determinantes (55,56\%). Sin embargo, el porcentaje de sensibilización para los demás pólenes ana- 
lizados resultó siempre menor para el grupo de pacientes hispanos frente a los pacientes españoles $(\mathrm{p}=0,005)$ (Tabla 3). La incidencia de sensibilización a polcalcina fue muy baja en toda la muestra. Sólo 4 pacientes, 2 españoles, 1 hispano y 1 de Europa del Este, estaban sensibilizados frente a este panalér- geno. Por contra, el 44,1\% de los españoles presentaban sensibilización a profilina, frente a sólo el 4,88 de los hispanos $(\mathrm{p}<0,0001)$, aunque la existencia de sensibilización a este panalérgeno no implicaba en la mayoría de los casos la presencia de síntomas con la toma de frutas u otros vegetales.

Tabla 3. Sensibilizaciones frente a alérgenos según grupos poblacionales.

\begin{tabular}{lrrrrr}
\hline & $\begin{array}{c}\text { Españoles } \\
(\mathrm{n}=33)\end{array}$ & $\begin{array}{c}\text { Europa Este } \\
(\mathrm{n}=10)\end{array}$ & $\begin{array}{c}\text { Hispanos } \\
(\mathrm{n}=41)\end{array}$ & $\begin{array}{c}\text { Árabes } \\
(\mathrm{n}=9)\end{array}$ & p \\
\hline Pólenes & & & & & \\
\hline \multicolumn{1}{c}{ Gramíneas } & $28(84,4 \%)$ & $6(60 \%)$ & $30(73,1 \%)$ & $5(55 \%)$ & NS \\
\hline Olivo & $19(57,6 \%)$ & $5(50 \%)$ & $10(24,3 \%)$ & $5(55,5 \%)$ & $0.005^{*}$ \\
\hline \multicolumn{1}{c}{ Platanus } & $5(15,1 \%)$ & $2(20 \%)$ & $2(4,8 \%)$ & $0(0 \%)$ & NS \\
\hline \multicolumn{1}{c}{ Artemisia } & $1(3,03 \%)$ & $0(0 \%)$ & $0(0 \%)$ & $0(0 \%)$ & NS \\
\hline Ácaros & $3(9,1 \%)$ & $2(20 \%)$ & $12(29,2 \%)$ & $5(55,5 \%)$ & $0,02^{*}$ \\
\hline Epitelios & $6(18,2 \%)$ & $0(0 \%)$ & $6(14,6 \%)$ & $1(11,1 \%)$ & NS \\
\hline Hongos & $3(9,1 \%)$ & $1(10 \%)$ & $1(2,4 \%)$ & $0(0 \%)$ & NS \\
\hline Profilina & $15(45,45 \%)$ & $2(20 \%)$ & $2(4,8 \%)$ & $2(22,2 \%)$ & $<0,0001^{*}$ \\
\hline Polcalcina & $2(6,06 \%)$ & $1(10 \%)$ & $1(2,4 \%)$ & $0(0 \%)$ & NS \\
\hline
\end{tabular}

*Valor de p comparando pacientes caucásicos con hispanos.

\section{DISCUSIÓN}

La incidencia de las enfermedades alérgicas ha aumentado en las últimas 2 décadas, en las naciones desarrolladas al tiempo que hemos asistido a un aumento del fenómeno migratorio hacia estos países. España constituye el décimo país del mundo por número de inmigrantes y se ha convertido en el primer destino europeo. Desde 1998, el colectivo de extranjeros ha aumentado en un $650 \%$, según datos del Instituto Nacional de Estadística (INE) ${ }^{9}$. A finales de 2008, la población extranjera constituía un $12,0 \%$ de la población empadronada en España, aunque en algunas zonas como la Comunidad de Madrid alcanzaba el $16,66 \%{ }^{10}$. De hecho, aunque algunos datos apuntan a las enfermedades alérgicas como las más prevalentes de entre las enfermedades crónicas en la población inmigrante, alrededor del $10,2 \%{ }^{11}$, en nuestro estudio el porcentaje de consultas por sospecha de alergia respiratoria en población extranjera alcanzó solamente el $7,23 \%$ del total de primeras visitas, si bien es posible que este hecho, en realidad, se deba a un periodo de inclusión corto. El grupo de Latinoamérica fue el que mayor número de pacientes incluía en consonancia con una mayor presencia de inmigrantes de este origen en nuestro medio, y es que, aunque en la Comunidad de Madrid, un $19,55 \%$ de los inmigrantes es de origen rumano y el $8,24 \%$ son de origen marroquí, considerados como un único conjunto de nacionalidades, los sujetos de origen hispanoamericano son ampliamente los más numerosos $(43,65)^{12}$. No se ha demostrado concluyente que la raza hispana favorezca el desarrollo de alergia ${ }^{13}$ y no podemos excluir que estos datos estén en relación con ser el grupo de extranjeros no comunitario más importante en España (28\%) ${ }^{9}$. 
En la actualidad se considera que la suma de factores genéticos y ambientales, es la responsable final de la aparición de las enfermedades alérgicas en individuos susceptibles $^{14}$. En este estudio, la tasa de antecedentes familiares, medida indirecta de la base genética para desarrollar enfermedades alérgicas, fue menor del $20 \%$, sin diferencias significativas entre los distintos grupos. En general, la alergia afecta menos a los grupos etarios mayores aunque en los últimos años, se observa un aumento de la expresión clínica de alergia "de novo" en sujetos de edad más avanzada ${ }^{15}$. Por ello, sería lógico pensar que existiera una base genética no expresada clínicamente por la mayor parte de los familiares de los sujetos del estudio, pero no podemos demostrarlo con estos datos.

Un hecho destacable es el tiempo de evolución desde el inicio de los síntomas, que es significativamente menor en el grupo de los pacientes extranjeros, particularmente en los hispanos. En éstos, es de apenas 4 años, frente a un tiempo medio de evolución de cerca de 8 años en los pacientes españoles. Podría, en parte, deberse a que, probablemente, el paciente extranjero reclama antes atención médica, pero creemos que el hecho de la mayor severidad de la rinitis y más temprana evolución hacia desarrollar asma bronquial (menos de 1 año en el caso de los pacientes hispanos frente al menos 3 , en los pacientes españoles) apunta a una rápida sensibilización a los aeroalérgenos, que deriva en una pronta expresión de sintomatología. Si el tiempo medio de residencia en España es de 84 meses ( 7 años), el debut clínico se produce en España en la mayoría de los casos y probablemente también la sensibilización a los alérgenos, hecho que se confirma al ser el polen de gramíneas el más relevante salvo en el caso de los pacientes árabes. Corroboraría la tendencia ya marcada en otros estudios previos, en los que el tiempo medio de evolución desde el momento de la inmigración oscilaba alrededor de los 5 años $^{16}$. Una de las hipótesis para justificar esta rápida sensibilización se basaría en un cierto carácter protector por parte de las infecciones por helmintos, que estos suje- tos sufrirían en sus países de origen, para modular la intensidad de la reacción alérgica respiratoria ${ }^{17,18}$. Al desaparecer este efecto al llegar a los países más desarrollados, acabarían por sensibilizarse frente a los alérgenos a los que se ven expuestos. Por ello, siendo el polen de gramíneas el más relevante en la zona centro de España, lo es también para la población analizada en este estudio, con la diferencia de los inmigrantes del norte de África, aunque, en este caso, la baja población muestra no da pie a realizar conclusiones cerradas. Si bien el haber elegido marzo puede introducir un sesgo, ya que, presumiblemente serán los alérgicos al polen los que consulten mayoritariamente en esa época, creemos que en nuestro estudio, la influencia estacional de la asistencia se esperaría igualmente en el grupo de pacientes españoles, por lo que no influiría significativamente en la comparación entre los dos grupos.

Además, es destacable la mayor incidencia de la sensibilización a la profilina en la población española, superior a lo publicado en algunas series ${ }^{19}$. Las profilinas son proteínas estructurales de las plantas que participan en la regulación del grado de polimerización de los filamentos de actina (componentes básicos del citoesqueleto) ${ }^{20}$. Actúan como panalérgenos siendo una de las principales responsables de la co-sensibilización entre pólenes y alimentos. Se supone que la sensibilización se produce por vía respiratoria al inhalar el polen, desarrollando posteriormente síntomas orales tras la exposición a la profilina de alimentos vegetales $^{21}$. Se baraja la posibilidad de que se asocie con mayor frecuencia a la sensibilización a polen de gramíneas ${ }^{22}$, si bien su trascendencia en la clínica todavía está por determinar ${ }^{19}$. En la población analizada en este estudio, la incidencia de alergia a alimentos es baja, por lo que, tal vez, la sensibilización se pudiera presentar previamente de modo subclínico, en relación con una exposición a mayores concentraciones ambientales de polen de gramíneas o durante un tiempo más prolongado de exposición. Para evaluar esta hipótesis, podría ser interesante desarrollar estudios prospectivos observacionales en estas po- 
blaciones y comprobar si, con el tiempo, manifiestan síntomas orales con la ingesta de algunas frutas.

Finalmente, de acuerdo a los datos reiteradamente recogidos en la literatura previamente ${ }^{23-24}$ los pacientes inmigrantes en este estudio presentan un peor nivel de control de la enfermedad. Se ha relacionado con factores culturales y económicos ${ }^{25}$, pero en nuestro caso, no se han estudiado suficientemente estas variables como para poder concluir su influencia en esta serie.

En resumen, presentamos los datos de un estudio multicéntrico que muestra diferencias significativas en el tiempo de evolución y los perfiles de sensibilización entre pacientes españoles e inmigrantes extranjeros con alergia respiratoria. Además, parecen observarse diferencias en la expresión clínica de la rinitis, que junto al peor control de la enfermedad asmática, puede condicionar una peor evolución clínica en el colectivo inmigrante.

\section{* ADDENDUM}

El Grupo ESPLORA está compuesto por:

Dra. María Jesús Trujillo. Hospital del Tajo. Aranjuez. Madrid.

Dra. Angélica Feliu. Hospital del Tajo. Aranjuez Madrid.

Dra. Eloína González Mancebo. Hospital de Fuenlabrada. Madrid.

Dra. Mar Gandolfo. Hospital de Fuenlabrada. Madrid.

Dr. David González de Olano. Hospital de Fuenlabrada. Madrid.

Dr. Javier Ruiz-Hornillos. Hospital Infanta Elena. Valdemoro. Madrid.

Dr. Aythamy Henríquez. Hospital Infanta Elena. Valdemoro. Madrid.

Dra. Emma González. Hospital Infanta Cristina. Parla. Madrid.

Dra. Aurora Losada. Hospital Infanta Cristina. Parla. Madrid.

Dra. Sandra Bermejo. Hospital Infanta Cristina. Parla. Madrid.

Dra. Carmen Vidal. Hospital Infanta Cristina. Parla. Madrid.
Dr. Antonio Moreno. Hospital Virgen de la Luz. Cuenca.

Dr. Jesús González Cervera. Hospital de Tomelloso. Ciudad Real.

Dr. Benito Rodríguez Domínguez. Hospital de Tomelloso. Ciudad Real.

Dr. Fernando de la Torre. Alk-Abelló. Madrid.

Dra. Lucía Jimeno. Alk-Abelló. Madrid.

Dra. Anabel Huescar. Alk-Abelló. Madrid.

Dra. Beatriz Rodríguez Jiménez. Hospital Universitario de Getafe. Madrid.

Dr. Javier Domínguez Ortega. Hospital Universitario de Getafe. Madrid.

\section{BIBLIOGRAFÍA}

1. KRÄMER U, OpPERmann H, RANFt U, SchäFER T, Ring $\mathrm{J}$, BeHRENDT H. Differences in allergy trends between east and west Germany and possible explanations. Clin Exp Allergy 2010; 40: 289-298.

2. D’Amato G, Cecchi L, D'Amato M, Liccardi G. Urban air pollution and climate change as environmental risk factors of respiratory allergy: an update. J Investig Allergol Clin Immunol 2010; 20: 95-102.

3. Peden D, ReEd CE. Environmental and occupational allergies. J Allergy Clin Immunol 2010; 125 (2 Suppl 2): S150-60.

4. Masoli M, Fabian D, Holt S, Beasley R. The global burden of asthma: executive summary for the GINA Dissemination Commitee Report. Allergy 2004; 59: 469-478.

5. Rottem M, Szyper-Kravitz M, Shoenfeld Y. Atopy and asthma in migrants. Int Arch Allergy Immunol 2005; 136: 198-204.

6. Brozek JL, Bousquet J, Baena-Cagnani CE, BoninI S, CANONICA GW, CASALE TB et al. Global allergy and asthma european network; Grading of recommendations assessment, development and evaluation working group. Allergic rhinitis and its impact on asthma (ARIA) guidelines: 2010 revision. J Allergy Clin Immunol 2010; 126: 466-476.

7. GEMA 2009. Guía Española para el manejo del asma. J Investig Allergol Clin Immunol 2010; 20 (Suppl 1): S 9-18.

8. Vega JM, Badia X, Badiola C, López-Viña A, OlaGUibel JM, PiCADO C et al. Covalair Investigator Group. Validation of the Spanish version of the asthma control test (ACT). J Asthma 2007; 44: 867-872 
9. Instituto Nacional de Estadística. www.ine. es/prensa/np551.pdf

10. Informe demográfico de la población extranjera en la Comunidad de Madrid. Enero 2010. www.madrid.org

11. Esteban Vasallo E, Domínguez Berjón F, Astray Mochales G, Génova-Meleras R, Pérez-Sania A, SÁnchez Pecurra L et al. Prevalencia de enfermedades crónicas diagnosticadas en población inmigrante y autóctona. Gac Sanit 2009; 23: 548-552.

12. Informe de población extranjera empadronada en junio de 2010 en la Comunidad de Madrid. Consejería de empleo, mujer e inmigración. www.madrid.org

13. Smith K, WarholaK T, Armstrong E, Leib M, ReHFELD R, MALONE D. Evaluation of risk factors and health outcomes among persons with asthma. J Asthma 2009; 46: 234-237.

14. Gaffin JM, Phipatanakul W. The role of indoor allergens in the development of asthma. Curr opin allergy. Clin Immunol 2009; 9: 128-135.

15. Bом T, Pinto AM. Allergic respiratory diseases in the elderly. Respir Med 2009; 103: 1614-1622.

16. Lombardi C, Penagos M, Senna G, Canonica GW, Passalacqua G. The clinical characteristics of respiratory allergy immigrants in northern Italy. Int Arch Allergy Immunol 2008; 147: 231-234.

17. Liu P, Li J, Yang X, Shen Y, Zhu Y, Wang S et al. Helminth infection inhibits airway allergic reaction and dendritic cells are involved in the modulation process. Parasite Immunol 2010 ; 32 : 57-66.
18. Flohr C, Quinnell RJ, BRitton J. Do helminth parasites protect against atopy and allergic disease? Clin Exp Allergy 2009; 39: 20-32.

19. Asero R, Jimeno L, BARBer D. Preliminary results of a skin prick test-based study of the prevalence and clinical impact of hypersensitivity to pollen panallergens (polcalcin and profilin). J Allergy Clin Immunol 2010; 20: 35-38.

20. Fernández-Rivas M, Barber R, Rodríguez R, SALCEDo G. Alérgenos alimentarios. En: Peláez Hernández A, Dávila González I. Tratado de Alergología. Ergon Ediciones. Majadahonda (Madrid) 2007: 807-830.

21. RodríGuez JimÉnEz B. Hipersensibilidad a profilina de comienzo precoz. En: Libro de sesiones interhospitalarias de la Sociedad Madrid Castilla-La Mancha de Alergología e Inmunología Clínica. Curso 2005-2006. Luzán 5 S.A. de Ediciones. Madrid 2007: 121-136.

22. Barber D, de la Torre F, Feo F, Florido F, Guardia $\mathrm{P}$, Moreno $\mathrm{C}$ et al. Understanding patient sensitization profiles in complex pollen areas: a molecular epidemiological study. Allergy 2008; 63: 1550-1558.

23. Yinusa-Nyahkoon LS, Cohn ES, Cortes DE, Bokhour BG. Ecological barriers and social forces in childhood asthma management: examining routines of African American families living in the inner city. J Asthma 2010; 47: 701-710.

24. Bacon SL, Bouchard A, Loucks EB, Lavole KL. Individual-level socio-economic status is associated with worse asthma morbidity in patients with asthma. Respir Res 2009; 10: 125.

25. BRYANT-STEPHENS T. Asthma disparities in urban environments. J Allergy Clin Immunol 2009; 123: 1199-1206. 